\title{
A Telemetric, Gravimetric Platform for Real-Time Physiological Phenotyping of Plant-Environment Interactions
}

\author{
Ahan Dalal ${ }^{1}$, Itamar Shenhar ${ }^{1}$, Ronny Bourstein ${ }^{1}$, Amir Mayo ${ }^{1}$, Yael Grunwald ${ }^{1}$, Nir Averbuch ${ }^{1}$, Ziv Attia ${ }^{1}$, Rony \\ Wallach $^{1}$, Menachem Moshelion ${ }^{1}$ \\ ${ }^{1}$ Faculty of Agriculture, Food and Environment, The Robert H. Smith Institute of Plant Sciences and Genetics in Agriculture, The Hebrew University of \\ Jerusalem
}

\section{Corresponding Author}

Menachem Moshelion

menachem.moshelion@mail.huji.ac.il

\section{Citation}

Dalal, A., Shenhar, I., Bourstein, R.,

Mayo, A., Grunwald, Y., Averbuch, N.,

Attia, Z., Wallach, R., Moshelion, M. A

Telemetric, Gravimetric Platform for

Real-Time Physiological Phenotyping of Plant-Environment Interactions. J. Vis. Exp. (162), e61280, doi:10.3791/61280 (2020).

\section{Date Published}

August 5, 2020

DOI

$10.3791 / 61280$

URL

jove.com/video/61280

\section{Abstract}

Food security for the growing global population is a major concern. The data provided by genomic tools far exceeds the supply of phenotypic data, creating a knowledge gap. To meet the challenge of improving crops to feed the growing global population, this gap must be bridged.

Physiological traits are considered key functional traits in the context of responsiveness or sensitivity to environmental conditions. Many recently introduced high-throughput (HTP) phenotyping techniques are based on remote sensing or imaging and are capable of directly measuring morphological traits, but measure physiological parameters mainly indirectly.

This paper describes a method for direct physiological phenotyping that has several advantages for the functional phenotyping of plant-environment interactions. It helps users overcome the many challenges encountered in the use of load-cell gravimetric systems and pot experiments. The suggested techniques will enable users to distinguish between soil weight, plant weight and soil water content, providing a method for the continuous and simultaneous measurement of dynamic soil, plant and atmosphere conditions, alongside the measurement of key physiological traits. This method allows researchers to closely mimic field stress scenarios while taking into consideration the environment's effects on the plants' physiology. This method also minimizes pot effects, which are one of the major problems in pre-field phenotyping. It includes a feed-back fertigation system that enables a truly randomized experimental design at a field-like plant density. This system detects the soil-water-content limiting threshold $(\theta)$ and allows for the translation of data into knowledge through the use of a real-time analytic tool and an online statistical resource. This method for the rapid 
and direct measurement of the physiological responses of multiple plants to a dynamic environment has great potential for use in screening for beneficial traits associated with responses to abiotic stress, in the context of pre-field breeding and crop improvement.

\section{Introduction}

Ensuring food security for a growing global population under deteriorating environmental conditions is currently one of the major goals of agriculture research ${ }^{1,2,3}$. The availability of new molecular tools has greatly enhanced crop-improvement programs. However, while genomic tools provide a massive amount of data, the limited understanding of actual phenotypic traits creates a significant knowledge gap. Bridging this gap is one of the greatest challenges facing modern plant science ${ }^{4,5,6}$. To meet the challenges that arise in the process of crop improvement and minimize the genotype-phenotype knowledge gap, we must balance the genotypic approach with a phenocentric one ${ }^{7,8}$.

Recently, various high-throughput phenotyping (HTP) platforms have made possible the nondestructive phenotyping of large plant populations over time and these platforms may help us to reduce the genotype-phenotype knowledge gap $6,8,9,10$. HTP screening techniques allow the measurement of traits in massive numbers of plants within a relatively short period of time, thanks to robotics and conveyor belts or gantries used to move the plants or sensors (respectively), as opposed to hand-operated techniques based on gas exchange or photography. Nevertheless, the massive amounts of data produced by HTP systems present additional data-handling and analytical challenges ${ }^{11,12}$.

Most of these HTP platforms involve the assessment of phenotypic traits through electronic sensors or automated image acquisition ${ }^{13,14}$. Advanced field phenomics involve the deployment of proximal sensors and imaging technologies in the field, as well as a high-resolution, precise and large-population scale of measurement ${ }^{15}$. Sensor and image data need to be integrated with other multi-omics data to create a holistic, second-generation phenomic approach ${ }^{16}$. However, methodological advances in data acquisition, handling and processing are becoming increasingly important, as the challenges of translating sensor information into knowledge have been grossly underestimated during the first years of plant phenomics research ${ }^{13}$. However, the reliability and accuracy of currently available imaging techniques for in depth phenotyping of dynamic genotype-environment interactions and plant stress responses are questionable ${ }^{17,18}$. Moreover, the results from controlled environments are often very different than those observed in the field, especially when it comes to drought-stress phenotyping. This is due to differences in the situation the plants experience in terms of soil volume, soil environment and mechanical impedance due to declining soil moisture during drought stress. Therefore, results from controlled environments are difficult to extrapolate to the field $^{19}$. Finally, the entry price of image-based HTP systems is very high, not only due to the price of sensors, but also due to the robotics, conveyor belts and gantries, which also require higher standards of growth-facility infrastructure and significant maintenance (many moving parts working in a greenhouse environment).

In this paper, we present an HTP-telemetric phenotyping platform designed to solve many of the problems 
mentioned above. Telemetry technology enables the automatic measurement and transmission of data from remote source(s) to a receiving station for recording and analysis. Here, we demonstrate a nondestructive HTP. telemetric platform that includes multiple weighing lysimeters (a gravimetric system) and environmental sensors. This system can be used for the collection and immediate calculation (image-analysis is not needed) of a wide range of data, such as whole-plant biomass gain, transpiration rates, stomatal conductance, root fluxes and water-use efficiency (WUE). The real-time analysis of the big data that is directly fed to the software from the controller in the system represents an important step in the translation of data into knowledge ${ }^{14}$ that has great value for practical decision-making, substantially extending the knowledge that can be acquired from controlled environment phenotyping experiments, in general, and greenhouse studies of drought stress, in particular.

Other advantages of the telemetry platform are its scalability and ease of installation and its minimal growth-facility infrastructure requirements (i.e., it can be easily installed in most growth facilities). Moreover, as this sensor-based system has no moving parts, maintenance costs are relatively low, including both the entry price and long-term maintenance costs. For example, the price of a 20-unit gravimetric system, including the feedback fertigation system for each plant, meteorological station and software, will be similar to the price of one portable gas-exchange system of a leading brand.

Rice (Oryza sativa L.) was used as a model crop and drought was the examined treatment. Rice was chosen as it is a major cereal crop with wide genetic diversity and it is the staple food for over half of the world's population ${ }^{20}$. Drought is a major environmental abiotic stress factor that can impair plant growth and development, leading to reduced crop yields ${ }^{21}$. This crop-treatment combination was used to demonstrate the platform's capabilities and the amount and quality of data that it can produce. For more information regarding the theoretical background for this method, please see 22 .

\section{Protocol}

In this protocol, we referred to $4 \mathrm{~L}$ pots loaded on $20 \mathrm{~cm} \mathrm{x}$ $20 \mathrm{~cm}$ scales, with each pot containing one plant. The same protocol is easily scalable and can be used with much bigger pots (up to $25 \mathrm{~L}$ loaded on $40 \mathrm{~cm}$ x $40 \mathrm{~cm}$ scales, with only a linear adaptation to the protocol measures) and several plants per pot. Thus the protocol can be easily adapted for plants of many types and sizes. Please refer to Figure 1 and Figure 2 for the system components.

\section{Prepare the pots for the experiment}

1. Insert the soil filter. Spread the nylon mesh (net) on top of the whole pot and place the net-holder on top of the net. With a hand, slowly push the net holder half way down the inside of the whole pot. Make sure that the net remains uniformly spread as it is pushed down between the two pots.

2. Insert the fiberglass stick (pole) between the two pots and push it all the way down to the bottom of the whole pot, making sure that it is on the outer side of the net as well and does not push the net.

3. Before pushing the net holder all the way down, push the net down by hand from inside the pot and adjust it so that it is spread uniformly and tightly over the bottom of the pot once the net holder has been fully inserted (Figure $\mathbf{2 C l}$ ).

4. Slide the gasket ring from the bottom of the pot set-up described above, a third of the way up the side of the 
pot. Make sure that the slits of the ring open toward the bottom of the pot (Figure 2CII).

5. Repeat steps 1.1-1.4 for all of the experimental pots before continuing on to the next step. Randomize the location of the plants (Figure 2D; in either a randomized block design or a fully random design) using the Array Randomizer application.

NOTE: To download the free program and for more information, please see the link: https://drive.google.com/open? id=1y4QbTpxRK5Lx430xzu1RFdrlcL8pz_1q).

6. Label the pots according to their locations in the array inside the greenhouse. For example, the label "B10D" corresponds to a pot located on Table B in Column 10 and Row D. Prepare three additional pots for each table for soil-water-content measurements (please see Section 7.1).

\section{Grow the plants}

1. Choose the growing (potting) medium that best suits the experiment. Choosing the right medium for the experiment is crucial and the correct choice depends on several factors (see Discussion). For first-time users, we strongly recommend using a porous, ceramic, smallsized medium. Please refer to Table 1 and Table 2 for more information to help choose the right medium for the experiment.

2. Germinate the seeds in cavity trays with the desired potting medium. If possible, do this inside the same greenhouse to be used for the main part of the experiment, in order to acclimate the plants to the environmental conditions inside that greenhouse.
3. If the seedlings were not germinated in trays, transplant them into cavity trays containing the potting medium. Plant one seedling in each cavity and let it grow until its roots are dense enough to take the shape of the cavity (root-soil plug).

4. Leave 5-7 cavities without seedlings for soil weight measurements (only potting medium; Figure 3). For more information, please see Section 5.9.

\section{Improve the signal-to-noise level}

NOTE: The following steps improve the quality of the measurements and reduce the noise levels.

1. Calibrate the lysimeter.

1. Use a spirit level to check that all of the lysimeters are level and then start the weight calibration process. Use two standard weights (1-10 kg). Perform the calibration while the green container, including all plugs, is on the load cell.

2. Put the first (lighter) calibration weight on each load cell.

3. In the operating software, go to the Calibration tab and choose the weight for the first point. Then, select the load-cell position where the weight was placed and click Get point1 (Supplementary Figure 1A). This step can be applied on several load-cells simultaneously.

4. Repeat for the second weight and click Get point2.

\section{Click Apply calibration.}

2. Ensuring a sufficient quantity of plants with an appropriate size for the experiment

NOTE: The smaller the plant, the weaker its signal will be (e.g., weight of water transpired in a day versus the 
pot weight). The following steps will help to improve the signal-to-noise ratio.

1. Start the experiment when the plant transpires approximately $10 \%$ of the maximum pot water capacity.

NOTE: For example, if working with a sandy medium that contains approx. $1 \mathrm{~L}$ of water at pot capacity (see Table 2), start the experiment when the plants transpire approx. $100 \mathrm{~mL}$ per day. If working with a peat-based medium that holds about $2 \mathrm{~L}$ of water at pot capacity (see Table 2), start the measurements when the plants transpire about $200 \mathrm{~mL}$ per day.

2. Estimate the initial plant daily transpiration before loading it onto the system by measuring (manually) morning vs. evening weight differences in a few seedlings.

3. When working with small plants, put several plants in each pot (e.g., six Arabidopsis plants in one 3.9 $L \operatorname{pot}^{23}$, to reach the recommended minimal level of transpiration $)^{*}$.

\section{Setting up the experiment}

NOTE: The process of setting up the experiment is designed to take into account the weight of all the parts of the system, namely, the weight of the potting medium (including the soilwater weight at pot capacity) and the initial weight of the seedlings. Follow the steps below:

1. If possible, work with similar static components that have similar weights. Static weight components include pot sets, soil probes and other plastic parts.

2. To start a new experiment, open the operating software. Open the Experiments tab in the menu on the left side of the screen. Click on Create New or duplicate the experiment properties from a previous experiment by right-clicking on the desired experiment and choosing Duplicate. Rename the experiment (Supplementary Figure 1B).

3. Make sure that no unit is being used in a different experiment currently running in the system. Check that all of the plants in the Plants table match the experimental design. If not, change the table according to the design (please see Sections 5.18, 6 and Supplementary Figure 1C).

4. Start the experiment by clicking on the experiment name and then clicking Start.

5. Take manual measurements of the pre-prepared empty pots (double pot, net, stick and black gasket ring). If using parts that are similar to one another, the average weight of 10 of them will be sufficient.

6. Mix the potting medium thoroughly with some water, for at least $1 \mathrm{~h}$, so that it breaks down into homogeneous particles and is saturated, to ensure uniformity and homogeneity. For first-time users, we strongly recommend using a porous, ceramic, smallsized medium (see Table 1 and Table 2). As a second option, use coarse sand.

1. Use a mechanical mixer (e.g., a concrete mixer).

2. When a highly homogenous medium (i.e., industrial sand) is being used, skip step 4.6.1.

7. Fill all of the pots uniformly for the experiment with the appropriate potting medium (e.g., sand, soil or peat).

8. Insert a cast of a cavity mold (Figure 3B) that is similar in shape and size to the root-soil plug of the seedlings (from cavity tray) into the middle of the potting medium. Push it in completely. Tap the bottom of the pot against the floor 
a few times to make sure that the potting medium is well distributed in the pot. Repeat for all pots.

9. Water the pots well and rinse off the outside of the pots. Allow the pots to drain for 30 min before continuing on to the next step. Make sure that the pots drain freely. If the potting medium drains too slowly (e.g., dense peat), premix it with an airy substrate (e.g., perlite; please see also Table 1 and Table 2) to ensure faster drainage.

10. After the drainage has completely stopped, place all of the filled pots on the center of the lysimeter array (in the green containers that are already there) according to the experimental design (Figure 2A).

11. Check that the green containers are properly fitted into the load cell cover and not touching one another.

12. In the operating software, open the Experiment tab and select the Measure Components tab. Click on Measure object. Name the measurement "1st measurement" (Supplementary Figure 1D).

13. Place the irrigation drippers, probes and pot covers on each pot. Make sure that the lines for the multioutlet drippers and the probe cables are supported by their respective stands (attached to the units for each lysimeter scale; Figure 1E) before placing them in the pots. Make sure that all of the drippers, probes and covers are securely positioned.

14. Wait up to $3 \mathrm{~min}$ for a new measurement to be taken (data are collected automatically every $3 \mathrm{~min}$ ) and then open the Experiment tab. Select the Experiment tab and click the Experiment. Meta-tag this measurement to the "1 ${ }^{\text {st }}$ measurement" taken and name it "Static components" (Supplementary Figure 1E). Meta-tags are used when wanting to record a weight value that is determined by subtracting one measured value from another.

15. After making any necessary adjustments to the system, wait for a new data point to be recorded (every $3 \mathrm{~min}$ ) before taking the next measurement.

16. Check the Static Components column to confirm that the values recorded in the Plants table do not include outliers. If any of the weights recorded are too low or too high, check for any interference with the load cell (e.g., make sure that nothing is touching it) and then take a new measurement (after the system was still for $3 \mathrm{~min}$ ).

17. Click the Plants tab. Export the Plants table as a spreadsheet, add the average pot weight (from Step 4.5) to the measurement of the static components - "Tare weight". Save and upload the file (import tab).

18. Make sure that all of the drippers are securely inserted into the potting medium and to the pipe coming from the controller. Back in the operating software, in the Experiment tab, select Treatments Scenarios. Click Create New to make a new "Plan".

19. In the plan, choose the first step (create a new step if needed) and open it. Choose "Test" for Treatment and "Never" for Termination. In the step option, choose any treatment that is listed in the Irrigation Treatments tab above Experiments (Supplementary Figure 1F; please see also Step 4.21). Press the Apply tab.

20. Extract the Plants table as a spreadsheet, add "Plan" to the Treatment column and add "1" to the Step column. Save and upload the file.

21. Under the Irrigation Treatments tab, choose the "Test" treatment and set it to an irrigation time of 4-5 min [with the exact amount of time depending on the volumetric water content (VWC) of soil used] to enable drainage. 
Set the time 2 min ahead and go to the pots in the greenhouse. Other treatments can also be created. (See the detailed explanation in Step 7.4.)

22. Check visually that all of the drippers are working and that water is dripping out of the perforated drain plug of the green container.

23. In the experiment, change the irrigation treatment on Plan "X", Step 1 (please see step 4.19-4.20) to the desired irrigation treatment. Make sure that each night irrigation (with fertigation; see Table 3 for the fertigation components used) is divided into several short pulses (events) with substantial pauses between them (at least three events every night), to ensure that the soil reaches its field capacity before dawn.

24. Let the irrigation program run for 1 or 2 days to let the soil reach its field capacity and continue on to the next phase.

\section{Starting the experiment}

NOTE: The data collected at this stage will be used as reference values for the rest of the experiment. Therefore, it is important to follow the next steps carefully.

1. Repeat Steps 4.18 through 4.20. Alternatively, start the process in the early morning, not long after the latest irrigation step.

2. Check visually that all of the pots are irrigated and that excess irrigation liquid is dripping out of the perforated drain plug of the green bath.

3. Remove the green, unperforated plug (from the lowest orifice) of the green container and let the water drain out completely. Then, put the plug back in its place (Figure 1D). If working on "drainage 0" (i.e., with the bottom hole open/the hollow drainage plug connected on the lowest hole), skip this step.
4. In the operating software, open the tab for the experiment and go to Measure Components. Click Measure Object and name the measurement as "Cast-pre". Gently remove all of the casts from the pots and then wait $3 \mathrm{~min}$ for a new measurement to be recorded (Supplementary Figure 1D).

5. Click Measure Object, name it "Cast-post" and metatag the measurement to "Cast-pre". The option will automatically calculate the difference between the two measured values and give the cast weight to verify the weight sensitivity.

6. Check the weight values in the Plants table. The difference between the "Cast-post" measurements should be no more than 20 or $30 \mathrm{~g}$.

7. To measure the weight of the wet soil, in the operating software, go to the Measure Components tab in the experiment and select the Measure Soil Wet Weight option. Take the measurement by clicking OK when asked. Check the Soil Wet Weight measurements in the Plants table of yotheur experiment. The weight will appear in the "Soil Wet Weight" column (Supplementary Figure 1D,G).

8. If some of the measurements seem to fluctuate inappropriately, please do the following:

1. Confirm that each pot is positioned correctly and is not touching any neighboring pot(s).

2. Disconnect the first controller on the table from the electricity (the rest of the controllers are serially connected to one another and thus will shut down as well) for 2 min and then reconnect it.

9. Measure manually the average weight of a few (5-10) cavities with potting medium (from Step 2.3) without 
seedlings (soil plug). [Make sure that the soil plugs are well irrigated (i.e., to field capacity after drainage) before manual measurements.] In the Measure Components tab, press Set Seedling Bulk-Soil Weight and fill in the average weight (Supplementary Figure 1D).

10. Click Measure Plant Initial Weight. This first measurement is a reference point before planting (Supplementary Figure 1D).

11. Make sure that the seedlings in the cavity trays are well irrigated (i.e., to field capacity after drainage). Gently pull the seedlings with their root-soil plug from the cavities, making sure not to injure them, and place them carefully into the cavities made by casts in the pots, according to the experiment design. It is preferable to transfer the plants at dawn or dusk, in order to minimize the stress to the plants (i.e., to minimize wilting).

12. Wait for $3 \mathrm{~min}$. Click Measure Plant Initial Weight again. This second measurement is the plant initial weight. Meta-tag the measurement to the first one (the reference point). The software will calculate the difference between the two measurements and subtract the Seedling BulkSoil Weight. The result is the plant initial weight.

13. Check the measured values in the Plants table of the experiment to make sure that they fall within a reasonable and logical range (Supplementary Figure 1C).

14. Saturate the soil by repeating Steps 4.18 through 4.20 .

15. Make sure that all of the pots are draining properly. If not, repeat the saturation process. Wait $30 \mathrm{~min}$ for the drainage to cease. (See also Table 1 regarding the correct choice of potting medium.)

16. Under the Measure Components tab, click Measure Reserved Water Weight (Supplementary Figure 1D).
17. Extract the Plants table as a spreadsheet, subtract the measured Plant Initial Weight and Seedling Bulk-Soil Weight from the reserved water weight measurement (the "Reserve Water Inventory" column). Upload the file (Supplementary Figure 1C).

18. Confirm that the time period during which daily transpiration will be recorded is appropriate for the goals of the experiment. Fill in the values in the experiment general tab as appropriate for the project (Supplementary Figure 1H).

1. Fill in zero hour: The time at which the software will check whether it needs to move to the next step in the treatment scenario.

2. Fill in daily transpiration values: Daily transpiration is calculated as the difference between two weight windows during the day, for all days. The daily transpiration start time is the time at which the software will begin to measure the average weight.

19. Monitor the plants for 1-2 days before starting a new experiment (duplicate and rename the experiment).

\section{Change the Plants Table}

1. Extract the Plants table as a spreadsheet and change the table according to needs. Do not change the Plant IDs, Names or Positions. Save and upload the file.

2. Labeling (grouping) columns: To present or analyze (please see Step 8) grouped plants based on common labels (e.g., treatment, line), add a new column and label starting with \# (e.g., \#Treatment). In this column, make a notation for each plant (e.g., for "\#Treatment" label, mark the plants as drought, control, etc.; Supplementary Figure 2). 
NOTE: The protocol presented above is the most advanced and comprehensive protocol for this system. However, first-time users may want to start with the simplified protocol (see Supplementary MS). The simplified protocol yields information about fewer traits and may lead to higher noise levels. But, at the same time, it provides a way to more easily become acquainted and familiar with the most important experimental procedures, hardware and software.

\section{Run the experiment}

1. Calculate the soil gravimetrical water content/soil water content (SWC value).

NOTE: Gravimetric soil water content is different from volumetric soil water content (VWC).

1. The SWC value is the ratio between the dry weight of the soil and the wet weight of the soil. To calculate SWC, use the three extra soil-filled pots (Step 1.3) without plants that were previously prepared and placed on a side table inside the greenhouse for a few days and irrigated regularly. Weigh the wet soil in an aluminum tray in the early morning, as soon as possible after the last irrigation event.

2. Dry the aluminum tray with the soil in an oven (at 105 ${ }^{\circ} \mathrm{C}$ ) for 4-5 days. Verify that the soil is completely dry by taking two consecutive weight measurements at least $60 \mathrm{~min}$ apart. If the weights are identical, the soil is indeed dry and the last measurement can be recorded as the dry soil weight.

3. In the operating software, go to Measure Components and click on the Calculate Soil Dry Weight tab. Fill in the soil wet and dry weights for each sample, click Apply and Finish (Supplementary Figure 3).
2. Alternatively, manually calculate SWC using equation shown below.

$$
\mathrm{SWC}=\frac{\text { Wet weight }- \text { Dry weight }}{\text { Dry weight }}
$$

3. Average the two SWC measurements taken manualy from at least three pots. Select the Measure Components tab and click on Calculate Soil Dry Weight the $\theta g[g / g]$ value, click Apply and Finish. The soil dry weights of all of the experiment pots will be calculated automatically by the software (assuming that all of the pots in the experiment contain the same medium; Supplementary Figure 1D and Supplementary Figure 3).

4. Apply the irrigation treatments. Irrigation scenarios can be applied by composing a step-by-step treatment plan.

1. To compose a new irrigation treatment plan, go to Irrigation Treatment, click on Create New, and name the new treatment. Open the specific treatment in the list of irrigation treatments and click the on the default "00:00".

NOTE: In the main window (Supplementary Figure 4A), "Time" indicates the time the valve will open (i.e., the beginning of the irrigation treatment). "Valve" is the valve to be opened (A or B, depending on the valve that is connected to the desired solution). "Command Type" indicates the type of data that will be used to determine when the valve will be closed:

1. By Time - How many seconds the valve will be open.

2. By Weight - The weight gain/water (in grams) to be added to the pot via irrigation. 
3. By Transpiration - Irrigation can be applied differentially to each pot based on the transpiration of each individual plant over the previous day. The user can decide what percentage of the previous day transpiration will be applied during irrigation. (Under the wellirrigated condition, it is suggested to give the plant more than $100 \%$, in order to wash the soil and compensate for plant growth.) Droughttreated plants should be given less water, with exact volumes based on the desired drought stress rate.

4. By Sensors - Irrigation can be applied according to a sensor reading, such as apparent dielectric permittivity (which can be used to determine the VWC). Select the sensor type, the desired parameter and the desired parameter value.

2. All possibilities include a Time Out option that will close the tap even if the set conditions were not reached. Set the Time Out for a period longer than the set conditions.

3. After defining the irrigation treatments for the experiment, open the desired experiment in the list of experiments, open Treatment Scenario, open default Plan and select the first step (Supplementary Figure 4B).

4. In Treatment, choose an irrigation treatment from the list. Then, in Termination, choose the appropriate condition to stop the current step and move on to the next one.

5. After selecting an irrigation scenario, open the experiment's Plants table (Supplementary Figure
2) and input the "Treatment" and "Step" for each plant. "Treatment" is the name of the treatment scenario and "Step" is the event number within the treatment scenario.

5. Plan a drought treatment.

1. Each individual plant has a unique transpiration rate based on its size and location in the greenhouse. To enable a standard drought treatment (i.e., similar drying rate for all pots during the treatment), plan a drought scenario and control it via the system's feedback-irrigation tool (Supplementary Figure 5).

\section{Analyze the data using data analysis software}

1. Open the Data Analysis software (e.g., SPAC Analytics). Click on the top right corner to select Control system and the name of the experiment (Supplementary Figure 6A). In the column of the left side of the screen, select Experiments (Supplementary Figure 6B) and type the name of the experiment in the Name bar under the Search section. The name of the experiment will appear below the Search section, in the Experiments section (Supplementary Figure 6C). Click on the experiment to open the Info and Plants sections (Supplementary Figure 6D).

2. In the Info section, edit the WUE start and WUE end dates for a period of at least 3 (preferably more) days before the start of the drought treatment and then click Update. The WUE and the $R^{2}$ value for every pot will appear in the Plants section. Choose to exclude any scale with a negative WUE value or an $R^{2}$ value of less than 0.5 by clicking on the "eye" symbol under the Active column, which will then turn red. This will exclude the selected scale (plant) from all further calculations. The data can 
be exported by clicking on the Export Data button in the Plants section (Supplementary Figure 6D).

3. In the column on the left side of the scree, click on Analysis. Different subsections will then appear: Graph viewer, Histogram, T-test, ANOVA and Piecewise linear curve.

4. Click on Graph viewer. In the Filters section, set the dates for the experiment.

1. Click on Labels (please see Step 6) to select the combination of experimental groups (genotype) and treatment(s). Automatically, all of the pots in the selected group will appear in the Plant subsection. In that subsection, deselect any pots (plants) by clicking on them. Up to two different parameters of choice can be selected at one time as the " $Y 1$ parameter" and "Y2 parameter". Finally, click on Show Graph (Supplementary Figure 5).

2. A line graph of the values of the selected parameter will appear in the Graph Viewer window for each plant. Remove data from individual plants or add to the graph by clicking on their legend symbols on the right of the graph. In the top right corner, there are also options for exporting the data as a spreadsheet and for enlarging the Graph Viewer window to fill the full screen (this raw data download function is relevant to all other windows). More options to modify the graph will appear if the cursor is moved to the top right corner of the screen (Supplementary Figure 5).

5. The histogram module presents the distribution of a single trait in and between populations for a given time period. To use this module, click on Histogram.
1. In the Filters section, set the date and time, parameter, labels and plants as explained in step 8.4.1. Select multiple labels (groups) by clicking on the + symbol. Finally, click on Show Graph (Supplementary Figure 7).

2. The histogram will appear in the Histogram section, in which there is the option to change the "Bins" and "Date" at the top of the screen. In the top right corner, there are various options as described in step 8.4.2. In the Location Diagram section, the actual location of the plants on the experimental table and their respective trait values can be seen (Supplementary Figure 7).

6. Click on T-test. To statistically compare the means of any measured trait of two groups, enter the dates, labels, plants and parameters in the "T-test Parameters" section, as explained in step 8.4.1.

1. Set the range of hours to calculate the average values of the data points within the time period of interest (the default is a continuous 24h presentation). Finally, click on Show Graph (Supplementary Figure 8).

2. Two windows will appear on the right side of the screen. The top one is the Graph Viewer section for all of the plants selected from both groups. Below that window is the T-test section, in which will appear the comparison of the two groups as the $t$ test of the physiological parameter selected. Levels of significance can be adjusted by changing the $\alpha$ value in the top left corner of the T-test section. A red dot will appear under values that are significantly different. In the top right corner, view various options, 
as described in step 8.4.2 (Supplementary Figure 8).

7. Click on ANOVA. To statistically compare the means of any measured trait across more than two groups, enter the dates, labels, plants and parameters in the "Filters" section, as explained in step 8.5.1.

1. Select multiple labels (groups) by clicking on the + symbol (as in step 8.5). Set the range of hours. Finally, click on "SHOW GRAPH" (Supplementary Figure 9).

2. In the ANOVA section, use an ANOVA test (Tukey's HSD) to compare the physiological parameters of the different groups. Bars represent the standard errors $( \pm S E)$. In the top right corner of the screen, there are various options as described in step 8.4.2. Click on the line graph to view a bar-graph comparison for a particular day. Different letters indicate groups that are significantly different from one another (Supplementary Figure 9A).

8. Presenting the relationship between whole-plant transpiration kinetics or stomatal conductance and VWC is a more accurate way to compare the physiological responses of different plants to drought, as compared to a time-based approach. Present this relationship using the "Piece-wise Linear Curve" function.

1. Click Piecewise linear curve. Enter the dates, labels, plants and parameters (both the $\mathrm{x}$-axis and the $y$-axis) and then set the range of hours in the "Filters" section, as explained above.

NOTE: The "from" date should be as close as possible to the treatment start date.

2. Set the x-axis parameter to be VWC and the y-axis parameter as the physiological parameter of choice

(e.g., transpiration rate, stomatal conductance, etc.). Finally, click on Show Graph. In the "Filter" section, click on Select all recommendations and then click on Show Graph (Supplementary Figure 10).

NOTE: Other physiological parameters (e.g., normalized transpiration, transpiration rate, plant initial weight, stomatal conductance, root flux, etc.) and environmental parameters (e.g., temperature, relative humidity, etc.) are easily obtained via the SPAC software (e.g. Supplementary Figure 9C). For more information regarding the theoretical background of their calculations, please see Halperin et al. (2017).

\section{Representative Results}

The duration of the experiment was 29 days. The experiment was conducted in August, when the local weather is warm and stable and the days are long. Two different irrigation scenarios were used to demonstrate the capability of the phenotyping platform for comparing the physiological behavior of three different varieties of rice (i.e., Indica, Karla, and Risotto) in the presence of drought stress. There were two drought-stress treatments: (i) optimal irrigation [until each pot reached its pot capacity at night after irrigation (control)] and (ii) a drought that started 5 days after the experiment started, lasted for 14 days, and was followed by a 10-day recovery period (optimal irrigation, Days 19-29). For the sake of simplicity, not all of the varieties and groups are shown in the figures presented here. The results showed that the HTP-telemetric system can efficiently measure changes in atmospheric conditions, the soil and the physiology of the plants.

\section{Environmental conditions}


Environmental conditions [photosynthetically active radiation (PAR) and vapor pressure deficit (VPD)] were monitored throughout the experiment by an atmospheric probe. The collected data indicate that PAR and VPD remained similar over the different days and over the course of the day (Figure 4).

The VWC of the drought-treated pots was measured by soil probes throughout the experimental period. The VWC data collected from one drought-treated cv. Indica plant is plotted in Figure 5.

\section{Physiological parameters}

The daily transpiration gradually increased in all four treatments (Karla-control, Karla-drought, Risotto-control and Risotto-drought) during the first stage of the experiment, during which all of the plants were well-irrigated. Later, there was a reduction in transpiration that was associated with the drought period (Day 5 to Day 18) in the two water-deprived treatments. Subsequently, during the recovery period (from Day 18 onward), the daily transpiration increased again in the two water-deprived groups, but to a much lower level than that observed before the drought treatment (Supplementary Figure 9B).

The mean calculated plant weight (i.e., rate of plant weight gain) increased consistently among both the Karla-control and the Karla-drought treatments during the first stage of the experiment, when all of the plants received similar irrigation (Days 1-5). When the drought treatment was applied to the cv. Karla plants (Days 5-18), those plants stopped gaining weight and did not resume gaining weight until the recovery stage. At that point, there was an increase in weight that proceeded more slowly than what was observed for the control. In contrast, the weights of the Karla-control plants increased continuously throughout the experimental period (Figure 6). 

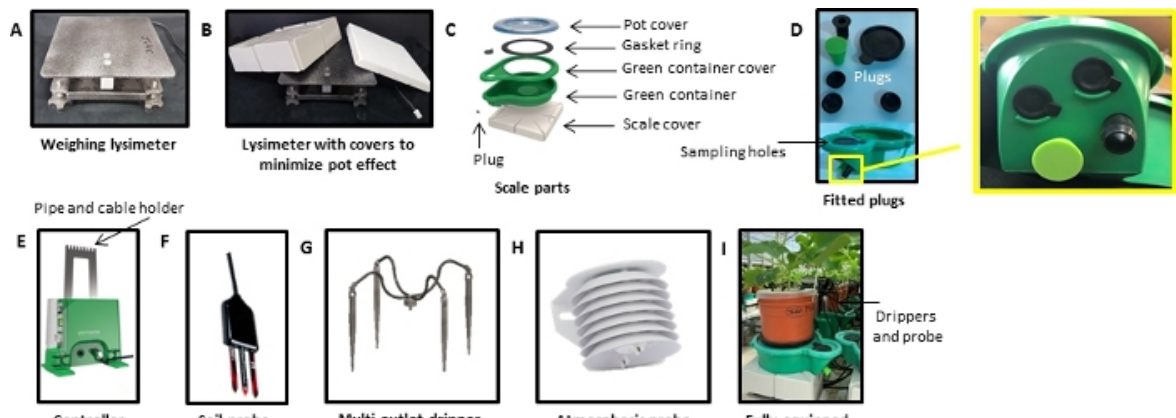

minimize pot effect

Scale parts Fitted plues

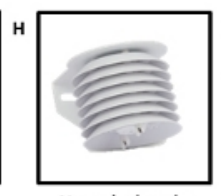

Atmospheric probe
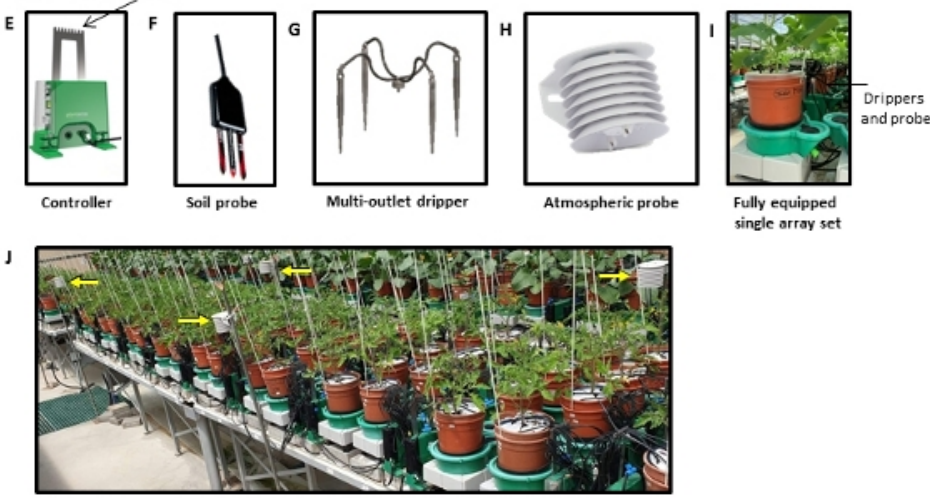

Fully equipped
single array set

Fully equipped array sets

Figure 1: Components and setup of the gravimetric phenotyping system.

(A) Weighing lysimeter. The lysimeter includes the load cell, which converts the mechanical load of an object into an electrical charge, and a metal platform that covers the upper and lower parts of the load cell, so that the object's weight can be properly measured. (B) The lysimeter is covered with a polystyrene block and a plastic cover for heat insulation. (C) Scale parts. A water reservoir (green container) is placed on the lysimeter cover to collect the liquid that drains from the pot. The green container is coupled to a green cover, which has a large round opening through which the pot is inserted. A black rubber gasket ring is attached to one side of the green cover and the pot is attached to the other side, to minimize water loss via evaporation from the container. The green cover has two sampling holes (small and big) above the drainage extension, which are sealed with rubber plugs. (D) Plugs. The container has a drainage extension with four holes (with plugs) at different heights, which can be used to adjust the water level in the container after the drainage through a particular hole stops (the reserve water volume). The desired water volume will depend on the plant species, the type of potting medium being used and the water requirements of the plants (i.e., estimated daily transpiration volume). (E) The control unit consists of a green rectangular box that contains the electronic controller and solenoid valves. There are holes through which fertigation solution can enter and exit the pots, as well as sockets for connecting the load cell and different sensors. Different treatments, such as different levels of salinity or different mineral compositions, can be applied via the fertigation solution. A metal stand is connected to the controller, to hold the pipes and cables and prevent them from touching the pots and adding weight. The other components required are $(\mathbf{F})$ soil probes (e.g., moisture, temperature and EC sensors - 5TE), optional (G) multi-outlet drippers (for fertigation and/or treatment applications) and $(\mathbf{H})$ atmospheric probes [for measuring vapor pressure deficit (VPD) and radiation]. (I) Fully equipped single array. (J) Fully equipped array in the greenhouse, 
yellow arrows pointing the atmospheric probes which enables the stomatal conductance normalization based on the local atmospheric conditions. Please click here to view a larger version of this figure.

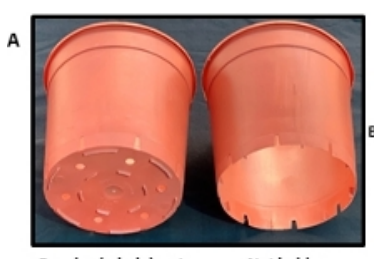

Net holder

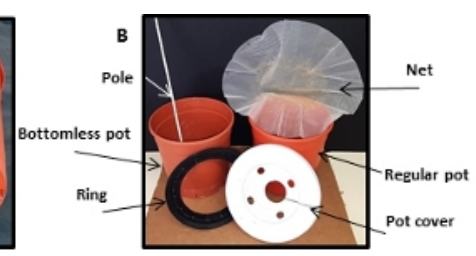

Pot cover
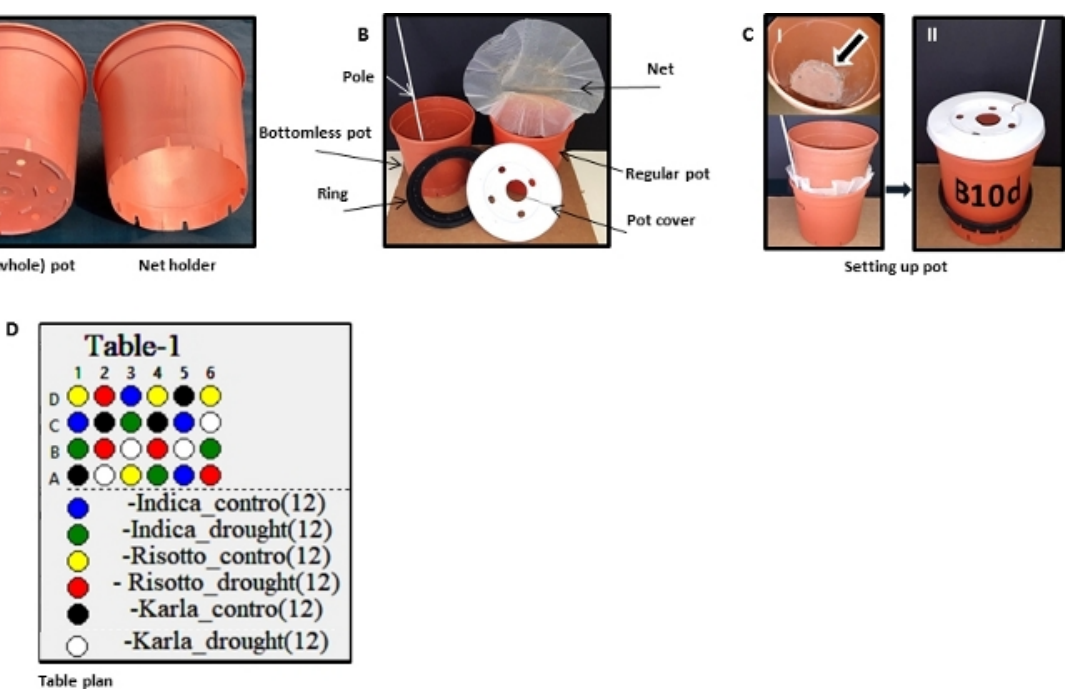

Figure 2: Parts required for a single pot set-up.

(A-C) The following components are needed: one $4 \mathrm{~L}$ pot, one $4 \mathrm{~L}$ pot with no bottom to serve as a net holder, one circular piece of nylon mesh (pore size $=60$ mesh) with a diameter double that of the bottom of the pot, one cover with designated holes for plant and irrigation drippers, one $60 \mathrm{~cm}$, white fiberglass stick (pole) and one black gasket ring. (D) Example of a table plan in which the pots have been randomized. In the greenhouse, each table had 1-18 columns and four rows, here we used 24 positions. However, the array structure can be easily adjusted to any shape based on the size of the own greenhouse. Please click here to view a larger version of this figure. 


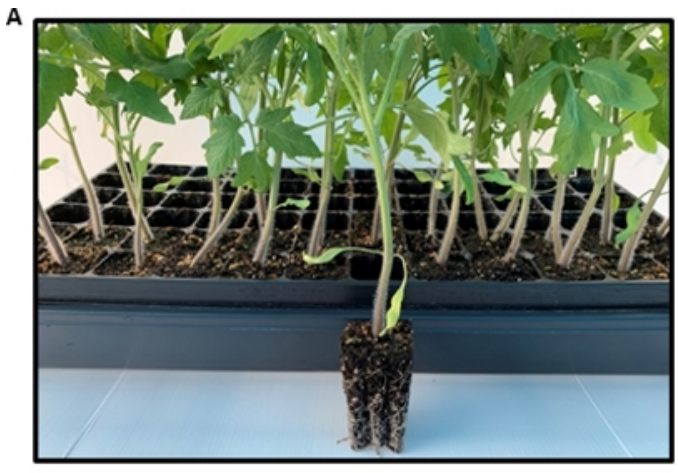

C

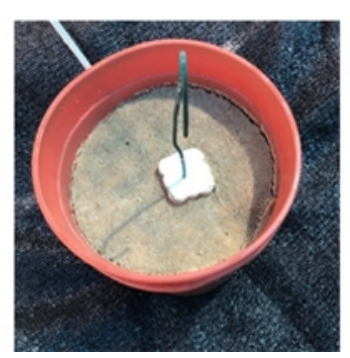

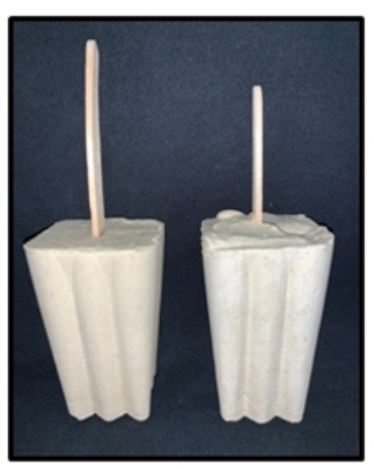

E

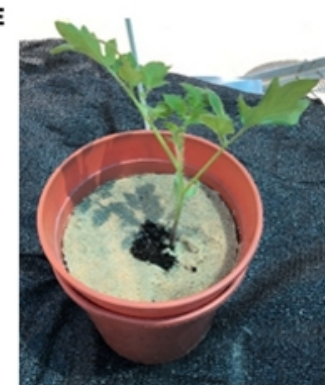

Figure 3: Pot set-up.

(A) Plants growing in cavity trays. (The tomato seedlings shown here are only an example; many other plant species could be grown in the same way). (B) Casts of molds for (C) creating cavities in the potting medium that will (D) closely fit the rootsoil plugs of the seedlings, to ensure the successful transplanting of $(E)$ the seedlings into the pots. Please click here to view a larger version of this figure. 


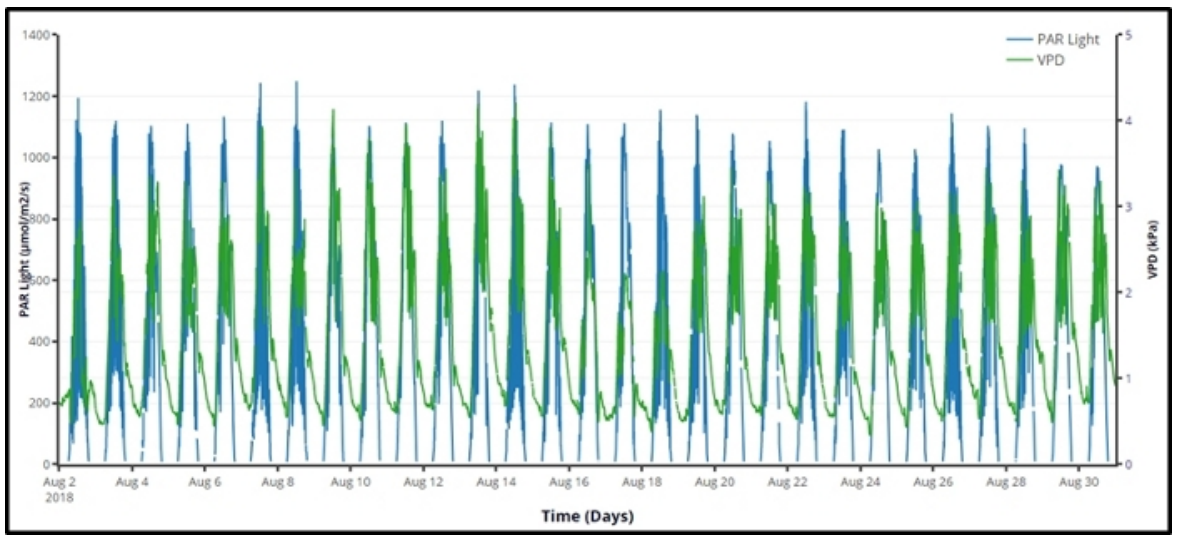

Figure 4: Atmospheric conditions over the course of the experiment.

The y-axis on the right shows the daily vapor pressure deficit (VPD) and the y-axis on the left shows the photosynthetically active radiation (PAR) over the 29 consecutive days of the experiment. This graph was produced by the Data Analysis software. Please click here to view a larger version of this figure.

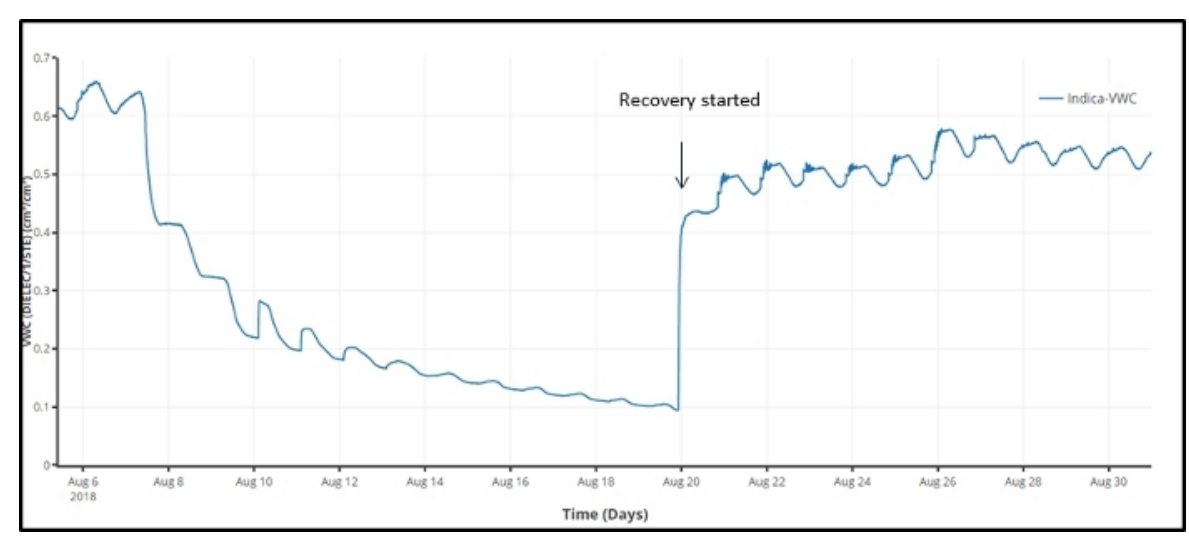

Figure 5: Volumetric water content (VWC) measured by a soil probe over the course of the experiment.

The data represent the VWC values for one $\mathrm{cV}$. Indica plant that was subjected to the drought treatment for the entire experiment period, including recovery. This graph was produced by the Data Analysis software. Please click here to view a larger version of this figure. 


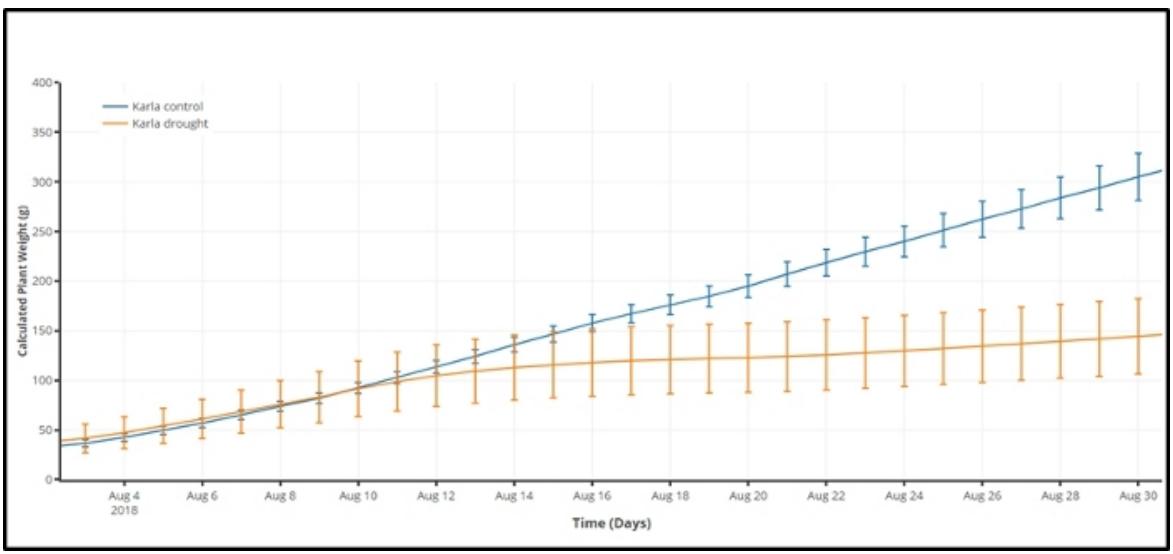

Figure 6: Whole-plant weights (means $\pm \mathrm{SE}$ ) over the entire experimental period for $\mathrm{cv}$. Karla under well-irrigated (control) and drought conditions.

Groups were compared using ANOVA (Tukey's HSD; $p<0.05$ ). Each mean \pm SE represents at least four plants. The graph and the statistical analysis were produced by the Data Analysis software. Please click here to view a larger version of this figure.

Supplementary Figure 1: Operating software windows for setting up an experiment. Please click here to download this figure.

Supplementary Figure 2: 'Plants' table as a spreadsheet; Operating software. Please click here to download this figure.

Supplementary Figure 3: Software window for calculating the soil dry weight; Operating software. Please click here to download this figure.

\section{Supplementary Figure 4: Software window for setting up an irrigation treatment; Operating software. Please click here to download this figure.}

Supplementary Figure 5: Data Analysis Graph Viewer window. In our experiment, we used three cultivars of rice (i.e., Indica, Karla, and Risotto) and two different irrigation scenarios, well-irrigated (control) and drought. The raw data revealed variation in the weight of the plants over the course of the experiment. Each line represents one plant/pot. During the day, the plants transpired, so the system lost weight, as can be seen in the slopes of the daily curves. The pots were irrigated every night to full capacity, as represented as the peaks in the curves. The irrigation event was followed by drainage of any excess water after the potting medium had been saturated. Initially, all plants were well irrigated (control). From 7 August 2018, half of the plants were subjected to a drought treatment. At the same time, the rest of the plants continued to receive optimal irrigation. Differential recovery was achieved by restoring the irrigation to the drought-treated plants, beginning on 20 August 2018 (allowing each plant to experience a similar degree of stress) and continuing through the experiment end. Please click here to download this figure.

The system's feedback-irrigation tool enables the user to design irrigation programs for each individual pot based on 
time, pot weight, data from a soil sensor (e.g., VWC) or plant transpiration over the previous day. Each plant can be irrigated individually in a customized manner based on its own performance. This differential irrigation minimizes the differences between the plants' soil water contents, so that all of the plants are exposed to a controlled drought treatment regardless of their individual water demands.

\section{Supplementary Figure 6: Data Analysis window for the} data analysis. Please click here to download this figure.

\section{Supplementary Figure 7: Data Analysis histogram} window. This figure shows a graphical representation of the distribution of daily-transpiration values in the three different rice cultivars (i.e., Indica, Karla, and Risotto) under wellirrigated (control) conditions. The bottom diagram represents a heat-map visualization of the plants daily transpiration based on the physical location of the pots on the table. Please click here to download this figure.

\section{Supplementary Figure 8: Data Analysis T-test window.} Lines represent the differences in daily transpiration (a fundamental and important physiological trait) between two rice cultivars (i.e., Karla and Risotto) under well-irrigated (control) conditions. The window shows the daily transpiration of the individual plants (top right) and a comparison of the means \pm SE of each group conducted using Student's $t$ test (bottom right). The statistical analysis was performed automatically by the software. The red dots represent significant differences between treatments according to the
Student's $t$-tests; $p<0.05$. Please click here to download this figure.

\section{Supplementary Figure 9: Data Analysis ANOVA window.}

(A) Graphical representation of the differences in daily transpiration between two rice varieties (i.e., Karla and Risotto) under well-irrigated (control) and drought conditions over the entire experimental period. The drought treatment was started 5 days after the experiment started. Clicking on any day will present the (B) Groups comparison using ANOVA (Tukey's HSD; $p<0.05$ ), here on AUG the 12th. Each mean \pm SE represents at least four plants. The same groups could be also presented as a (C) Continuous whole-plant transpirationrate (Means $\pm S E$ ) over the entire experimental period. The graphs and the statistical analysis were produced by the Data Analysis software. Please click here to download this figure.

\section{Supplementary Figure 10: Data Analysis piece-wise} linear curve window. This window shows the piece-wise linear curves of three rice cultivars (i.e., Indica, Karla and Risotto) under drought conditions. The software can perform a piece-wise linear fit analysis of the relationship between any physiological parameter (here, daily transpiration) and the calculated volumetric water content (VWC) of the plants subjected to the drought treatment. Please click here to download this figure.

Supplementary Materials. Please click here to download these materials.

\begin{tabular}{|c|c|}
\hline Medium & Description \\
\hline Coarse sand & $\begin{array}{r}\text { Silica sand 20-30 (upper and lower mesh screens through } \\
\text { which the sand was passed: } 0.841 \text { and } 0.595 \mathrm{~mm}, \text { respectively) }\end{array}$ \\
\hline Fine sand & $\begin{array}{r}\text { Silica sand 75-90 (upper and lower mesh screens through } \\
\text { which the sand was passed: } 0.291 \text { and } 0.163 \mathrm{~mm}, \text { respectively) }\end{array}$ \\
\hline
\end{tabular}




\begin{tabular}{|c|c|}
\hline Peat-based soil & Klasmann 686 \\
\hline Loamy soil (natural soil) & $\begin{array}{l}\text { Sandy loam soil taken from the top layer of a plot at the experimental } \\
\text { farm of the Faculty of Agriculture, Food and Environment, Rehovot, Israel }\end{array}$ \\
\hline Vermiculite & Vermiculite $3 G$ \\
\hline Perlite & Perlite 212 (Size range: $0.5-2.5 \mathrm{~mm}$ ) \\
\hline Compost & Bental 11 Potting soil \\
\hline Porous, ceramic, small-sized medium & $\begin{array}{l}\text { Profile Porous Ceramic } 20-50 \text { (upper and lower mesh screens through } \\
\text { which the ground ceramic was passed: } 0.841 \text { and } 0.297 \mathrm{~mm} \text {, respectively) }\end{array}$ \\
\hline Porous, ceramic, mixed-sized medium & Profile Porous Ceramic 50\% 20-50 mesh and 50\% 20-6 mesh, $0.841-3.36 \mathrm{~mm}$ \\
\hline
\end{tabular}

Table 1: Potting media. 


\begin{tabular}{|c|c|c|c|c|c|c|c|c|c|}
\hline $\begin{array}{c}\text { Soil media } \\
\text { type } / \\
\text { Parameters }\end{array}$ & $\begin{array}{c}\text { Coarse } \\
\text { sand }\end{array}$ & Fine sand & $\begin{array}{c}\text { Loamy } \\
\text { soil }\end{array}$ & Perlite & Vermiculite & $\begin{array}{l}\text { Porous } \\
\text { ceramic } \\
\text { mixed- } \\
\text { sized }\end{array}$ & $\begin{array}{c}\text { Porous } \\
\text { ceramic } \\
\text { small- } \\
\text { sized }\end{array}$ & $\begin{array}{c}\text { Peat- } \\
\text { based soil }\end{array}$ & Compost \\
\hline $\begin{array}{c}\text { Total water } \\
(\mathrm{TW}, \mathrm{ml})\end{array}$ & $\begin{array}{c}860 \pm \\
7.2(F)\end{array}$ & $\begin{array}{c}883.1 \pm \\
24(F)\end{array}$ & $\begin{array}{l}1076.3 \pm \\
35.9(E)\end{array}$ & $\begin{array}{r}1119.9 \\
\pm 8.5(\mathrm{E})\end{array}$ & $\begin{array}{c}1286 \pm \\
22.4(D)\end{array}$ & $\begin{array}{l}1503.6 \pm \\
15.4(C)\end{array}$ & $\begin{array}{c}1713 \pm \\
25.9(\mathrm{~B})\end{array}$ & $\begin{array}{r}1744.3 \\
\pm 8.2(\mathrm{~B})\end{array}$ & $\begin{array}{l}2089.6 \pm \\
61.6(\mathrm{~A})\end{array}$ \\
\hline $\begin{array}{c}\text { Volumetric } \\
\text { water } \\
\text { content } \\
(\mathrm{VWC} \\
\left.\mathrm{ml}^{3} / \mathrm{ml}^{3}\right)\end{array}$ & $0.26(F)$ & $0.27(F)$ & $0.33(E)$ & $0.35(E)$ & $0.4(D)$ & $0.46(\mathrm{C})$ & $0.53(\mathrm{~B})$ & $0.54(\mathrm{~B})$ & $0.65(\mathrm{~A})$ \\
\hline $\begin{array}{c}\text { Bulk } \\
\text { density } \\
(\mathrm{BD}, \mathrm{g} / \\
\left.\mathrm{cm}^{3}\right)\end{array}$ & $1.7(\mathrm{~A})$ & $1.6(\mathrm{~B})$ & $1.5(\mathrm{C})$ & $0.1(H)$ & $0.2(F)$ & $0.8(D)$ & $0.7(E)$ & $0.2(G)$ & $0.1(G)$ \\
\hline $\begin{array}{c}\text { Soil weight } \\
\text { stability } \\
\text { (SWS, g/d) }\end{array}$ & $\begin{array}{l} \pm 2.3 \pm \\
0.3(\mathrm{~B})\end{array}$ & $\begin{array}{l} \pm 4.3 \pm \\
0.3(\mathrm{~B})\end{array}$ & $\begin{array}{l} \pm 2.9 \pm \\
0.9(\mathrm{~B})\end{array}$ & $\begin{array}{l} \pm 14.9 \pm \\
0.7(\mathrm{~A})\end{array}$ & $\begin{array}{l} \pm 7.6 \pm \\
2.8(\mathrm{~B})\end{array}$ & $\begin{array}{l} \pm 1.3 \pm \\
0.1(\mathrm{~B})\end{array}$ & $\begin{array}{l} \pm 1.9 \pm \\
0.4(\mathrm{~B})\end{array}$ & $\begin{array}{l} \pm 6.7 \pm \\
0.8(\mathrm{~B})\end{array}$ & $\begin{array}{l} \pm 4.3 \pm \\
1.2(\mathrm{~B})\end{array}$ \\
\hline $\begin{array}{c}\text { Soil weight } \\
\text { stability } \\
\text { with } \\
\text { reserved } \\
\text { water in } \\
\text { the bath } \\
\text { (g/day; } \\
\text { please see } \\
\text { Section } \\
6.14)\end{array}$ & $3 \pm 0.4(B)$ & $\begin{array}{c}3.3 \pm \\
0.4(\mathrm{~B})\end{array}$ & $\begin{array}{c}3.2 \pm \\
1.2(B)\end{array}$ & $\begin{array}{c}6.3 \pm \\
0.5(A)\end{array}$ & $\begin{array}{c}2.7 \pm \\
0.8(\mathrm{~B})\end{array}$ & $\begin{array}{c}1.6 \pm \\
0.3(\mathrm{~B})\end{array}$ & $\begin{array}{c}1.9 \pm \\
0.3(\mathrm{~B})\end{array}$ & $\begin{array}{c}10.6 \pm \\
3(\mathrm{~A})\end{array}$ & $\begin{array}{c}1.5 \pm \\
0.3(\mathrm{~B})\end{array}$ \\
\hline $\begin{array}{c}\text { Pot } \\
\text { capacity } \\
\text { gravimetric } \\
\text { moisture }\end{array}$ & $0.18(G)$ & $0.23(\mathrm{G})$ & $0.23(G)$ & $3.79(\mathrm{C})$ & $3.0(D)$ & $0.74(F)$ & $0.99(E)$ & $4.25(\mathrm{~B})$ & $6.13(\mathrm{~A})$ \\
\hline
\end{tabular}




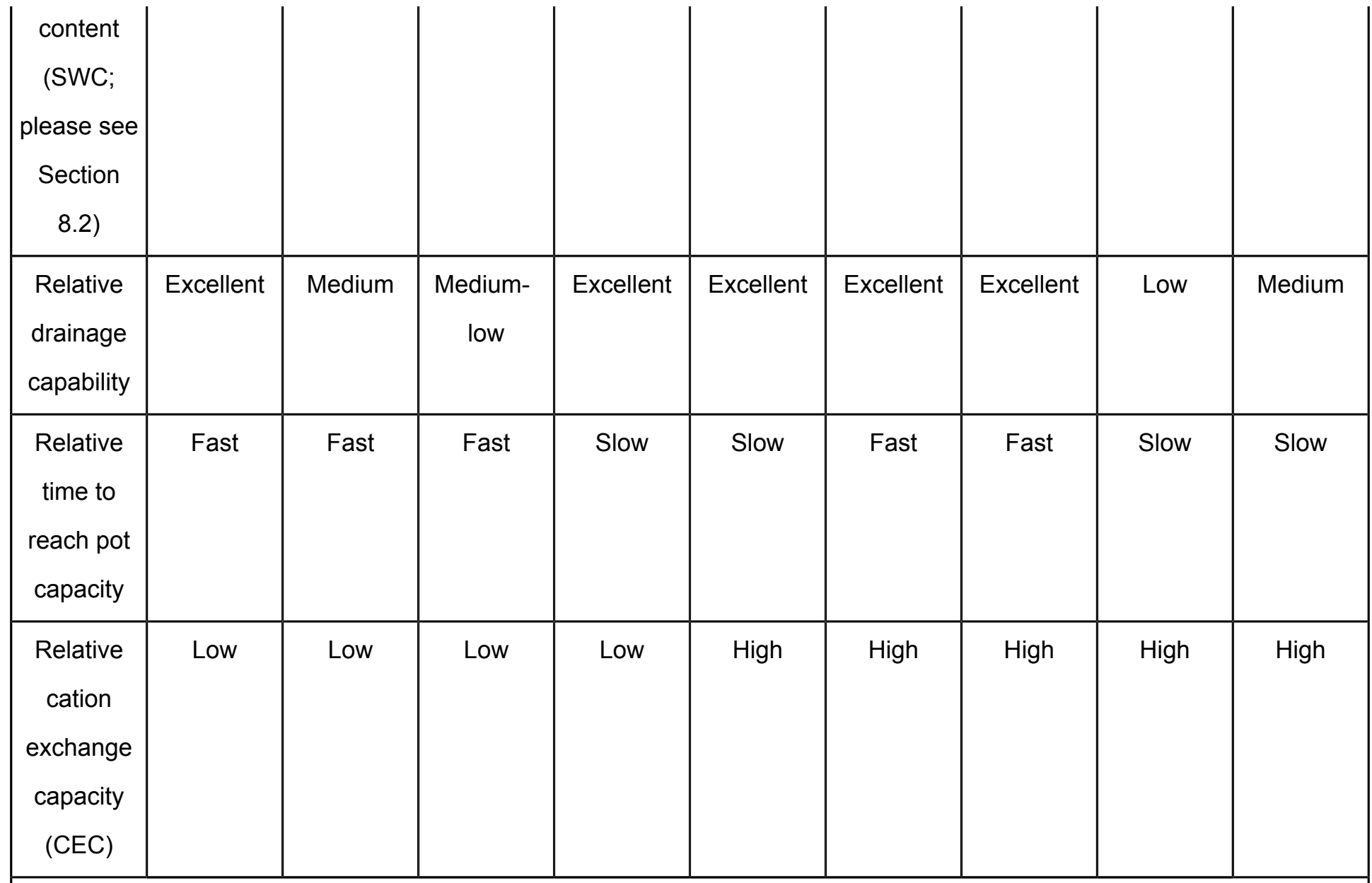

Compatibility with:

\begin{tabular}{|c|c|c|c|c|c|c|c|c|c|}
\hline $\begin{array}{c}\text { Root } \\
\text { washing } \\
\begin{array}{c}\text { (at the } \\
\text { end of the } \\
\text { experiment) }\end{array}\end{array}$ & ++ & ++ & + & ++ & + & ++ & ++ & - \\
\hline $\begin{array}{c}\text { Nutrient/ } \\
\text { biostimulant } \\
\text { treatment }\end{array}$ & ++ & ++ & - & ++ & + & + & + & - & - \\
\hline $\begin{array}{c}\text { Salinity } \\
\text { treatments }\end{array}$ & ++ & ++ & + & ++ & + & ++ & ++ & + & - \\
\hline $\begin{array}{c}\text { Accurate } \\
\text { neasurement } \\
\text { of growth } \\
\text { rates }\end{array}$ & ++ & ++ & + &,-+ & + & ++ & +++ & + & + \\
\hline
\end{tabular}




\begin{tabular}{|c|c|c|c|c|c|c|c|c|c|}
\hline $\begin{array}{l}\text { Physical } \\
\text { soil } \\
\text { structure } \\
\text { recovery } \\
\text { after } \\
\text { drought }\end{array}$ & +++ & +++ & ++ & + & - & +++ & +++ &,-+ & - \\
\hline \multicolumn{10}{|c|}{$\begin{array}{l}{ }^{*} \text { Total water }(T W, \mathrm{ml})=\text { soil wet weight (at pot capacity) - soil } \\
\text { dry weight. Volumetric water content (VWC) }=\text { TW/soil volume. }\end{array}$} \\
\hline \multicolumn{10}{|c|}{$\begin{array}{l}\text { Bulk density }(B D)=\text { soil dry weight/soil volume. Soil weight stability (SWS) = Average change in soil } \\
\text { wet weight over } 4 \text { consecutive days (medium at pot capacity with no plant after the last irrigation). }\end{array}$} \\
\hline
\end{tabular}

Table 2: General characteristics of 9 different potting media and their compatibility with the gravimetric platform.

The measurements were taken using 4-L pots filled with 3.2 L of medium at field capacity (pot capacity). Data are shown as means \pm SE. Different letters in the columns indicate significant differences between the media, according to Tukey's HSD test $(P<0.05 ; 3 \leq n \leq 5)$. 


\begin{tabular}{|c|c|c|}
\hline Fertigation components & Final concentration (ppm) & Final concentration (mM) \\
\hline $\mathrm{NaNO}_{3}$ & 195.8 & 2.3 \\
\hline $\mathrm{H}_{3} \mathrm{PO}_{4}$ & 209 & 0.000969 \\
\hline $\mathrm{KNO}_{3}$ & 271.4 & 2.685 \\
\hline $\mathrm{MgSO}_{4}$ & 75 & 0.623 \\
\hline $\mathrm{ZnSO}_{4}$ & 0.748 & 0.0025 \\
\hline $\mathrm{CuSO}_{4}$ & 0.496 & 0.00198 \\
\hline $\mathrm{MoO}_{3}$ & 0.131 & 0.00081 \\
\hline $\mathrm{MnSO} 4$ & 3.441 & 0.0154 \\
\hline Borax & 0.3 & 0.00078 \\
\hline $\mathrm{C} 10 \mathrm{H} 12 \mathrm{~N} 2 \mathrm{NaFeO}(\mathrm{Fe})$ & 8.66 & 0.0204 \\
\hline
\end{tabular}

The $\mathrm{pH}$ of the final irrigation solution from the dripper (after dilution with tap water) varied between 6.5 and 7.

Table 3: Fertigation components.

\section{Discussion}

The genotype-phenotype knowledge gap reflects the complexity of genotype $\mathrm{x}$ environment interactions (reviewed by $\left.{ }^{18,24}\right)$. It might be possible to bridge this gap through the use of high-resolution, HTP-telemetric diagnostic and phenotypic screening platforms that can be used to study whole-plant physiological performance and waterrelation kinetics 8,9 . The complexity of genotype $x$ environment interactions makes phenotyping a challenge, particularly in light of how rapidly plants respond to their changing environments. Although various phenotyping systems are currently available, most of those systems are based on remote sensing and advanced imaging techniques. Although those systems provide simultaneous measurements, to a certain extent, their measurements are limited to morphological and indirect physiological traits ${ }^{25}$.
Physiological traits are very important in the context of responsiveness or sensitivity to environmental conditions ${ }^{26}$. Therefore, direct measurements taken continuously and simultaneously at a very high resolution (e.g., $3 \mathrm{~min}$ intervals) can provide a very accurate description of a plant's physiological behavior. Despite those substantial advantages of the gravimetric system, the fact that this system has some potential disadvantages must also be taken into account. The main disadvantages result from the need to work with pots and in greenhouse conditions, which can present major challenges for treatment-regulation (particularly the regulation of drought treatments) and experimental-repeatability.

In order to address these issues, one should standardize the applied stresses, create a truly randomized experimental structure, minimize pot effects and compare multiple dynamic 
behaviors of plants under changing environmental conditions within a short period of time. The HTP-telemetric functional phenotyping approach described in this paper addresses those issues as noted below.

In order to correlate the plant's dynamic response with its dynamic environment and capture a complete, big picture of complex plant-environment interactions, both environmental conditions (Figure 4) and plant responses (Supplementary Figure 9B) must be measured continuously. This method enables the measurement of physical changes in the potting medium and atmosphere continuously and simultaneously, alongside plant traits (soil-plant-atmosphere continuum, SPAC).

To best predict how plants will behave in the field, it is important to perform the phenotyping process under conditions that are as similar as possible to those found in the field ${ }^{18}$. We conduct the experiments in a greenhouse under semi-controlled conditions to mimic field conditions as much as possible. One of the most important conditions is the growing or potting medium. Selecting the most suitable potting medium for the gravimetric-system experiment is crucial. It is advisable to choose a soil medium that drains quickly, allows for the rapid achievement of pot capacity and has a highly stable pot capacity, as those features allow for more accurate measurements by the gravimetric system. In addition, the different treatments to be applied in the experiment must also be considered. For example, treatments involving salts, fertilizers or chemicals call for the use of an inert potting medium, preferably one with a low cation-exchange capacity. Drought treatments applied to low-transpiring plant species would work best with potting media with relatively low VWC levels. In contrast, slow drought treatments applied to high-transpiring plants would

work best with potting media with relatively high VWC levels. If the roots are required for post-experiment analysis (e.g., root morphology, dry weight, etc.), the use of a medium with relatively low organic matter content (i.e., sand, porous ceramic or perlite) will make it easier to wash the roots without damaging them. For experiments that will continue for longer periods, it is advisable to avoid media that are rich in organic matter, as that organic matter may decompose with time. Please see Table 1 and Table 2 for more detailed information on this topic.

Field phenotyping and greenhouse phenotyping (pre-field) have their own objectives and require different experimental set-ups. Pre-field phenotyping assists the selection of promising candidate genotypes that have a high probability of doing well in the field, to help make field trials more focused and cost-effective. However, pre-field phenotyping involves a number of limitations (e.g., pot effects) that can cause plants to perform differently than they would under field conditions $^{18,27}$. Small pot size, water loss by evaporation and heating of the lysimeter scales are examples of factors in greenhouse experiments that may lead to pot effects ${ }^{18}$. The method described here is designed to minimize those potential effects in the following manner:

(a) The pot size is chosen based on the genotype to be examined. The system is capable of supporting various pot sizes (up to $25 \mathrm{~L}$ ) and irrigation treatments, which enables the examination of any type of crop plant.

(b) The pots and the lysimeter scales are insulated to prevent heat from being transferred and any warming of the pots.

(c) This system involves a carefully designed irrigation and drainage system. 
(d) There is a separate controller for each pot, to enable true randomization with self-irrigating and self-monitored treatments.

(e) The software takes into account the plants' local VPD in calculating the canopy stomatal conductance. Please see the multiple VPD stations localization in Figure 1J.

This system involves direct physiological measurements at field-like plant densities, which eliminates the need for either large spaces between the plants or moving the plants for image-based phenotyping. This system includes realtime data analysis, as well as the ability to accurately detect the physiological stress point $(\theta)$ of each plant. This enables the researcher to monitor the plants and make decisions regarding how the experiment is to be conducted and how any samples are to be collected over the course of the experiment. The system's easy and simple weight calibration facilitates efficient calibration. High-throughput systems generate massive amounts of data, which present additional data-handling and analytical challenges ${ }^{11,12}$. The real-time analysis of the big data that is directly fed to the software from the controller is an important step in the translation of data into knowledge ${ }^{14}$ that has great value for practical decision-making.

This HTP-telemetric physiological phenotyping method might be helpful for conducting greenhouse experiments under close-to-field conditions. The system is able to measure and directly calculate water-related physiological responses of plants to their dynamic environment, while efficiently overcoming most of the problems associated with the pot effect. This system's abilities are extremely important in the pre-field phenotyping stage, as they offer the possibility to predict yield penalties during early stages of plant growth.

\section{Disclosures}

The authors have nothing to disclose.

\section{Acknowledgments}

This work was supported by the ISF-NSFC joint research program (grant No. 2436/18) and was also partially supported by the Israel Ministry of Agriculture and Rural Development (Eugene Kandel Knowledge Centers) as part of the Root of the Matter - The Root Zone Knowledge Center for Leveraging Modern Agriculture.

\section{References}

1. Ray, D.K., Mueller, N.D., West, P.C., Foley, J.A. Yield Trends Are Insufficient to Double Global Crop Production by 2050. PLoS One. 8, e66428 (2013).

2. Food and Agriculture Organization of the United Nations. The future of food and agriculture: Trends and challenges. Rome. (2017).

3. Dhankher, O.P., Foyer, C.H. Climate resilient crops for improving global food security and safety. Plant, Cell \& Environment. 41, 877-884 (2018).

4. Chen, D. et al. Dissecting the phenotypic components of crop plant growthand drought responses based on highthroughput image analysis w open. Plant Cell. 26, 46364655 (2014).

5. Ubbens, J.R., Stavness, I. Deep Plant Phenomics: A Deep Learning Platform for Complex Plant Phenotyping Tasks. Frontiers in Plant Science. (2017).

6. Danzi, D. et al. Can High Throughput Phenotyping Help Food Security in the Mediterranean Area? Frontiers in Plant Science. (2019). 
7. Miflin, B. Crop improvement in the 21st century. Journal of Experimental Botany. 51, 1-8 (2000).

8. Dalal, A. et al. Dynamic Physiological Phenotyping of Drought-Stressed Pepper Plants Treated With "Productivity-Enhancing" and "Survivability-Enhancing" Biostimulants. Frontiers in Plant Science. (2019).

9. Moshelion, M., Altman, A. Current challenges and future perspectives of plant and agricultural biotechnology. Trends in Biotechnology. 33, 337-342 (2015).

10. Singh, A., Ganapathysubramanian, B., Singh, A.K., Sarkar, S. Machine Learning for High-Throughput Stress Phenotyping in Plants. Trends in Plant Science. 21, 110124 (2016).

11. Houle, D., Govindaraju, D.R., Omholt, S. Phenomics: The next challenge. Nature Reviews Genetics. 11, 855866 (2010).

12. Fiorani, F., Schurr, U. Future Scenarios for Plant Phenotyping. Annual Review of Plant Biology. 64, 267291 (2013).

13. Tardieu, F., Cabrera-Bosquet, L., Pridmore, T., Bennett, M. Plant Phenomics, From Sensors to Knowledge. Current Biology. 27, R770-R783 (2017).

14. Negin, B., Moshelion, M. The advantages of functional phenotyping in pre-field screening for drought-tolerant crops. Functional Plant Biology. (2017).

15. Gebremedhin, A., Badenhorst, P.E., Wang, J., Spangenberg, G.C., Smith, K.F. Prospects for measurement of dry matter yield in forage breeding programs using sensor technologies. Agronomy. 9, 65 (2019).
16. Roitsch, T. et al. New sensors and data-driven approaches-A path to next generation phenomics. Plant Science. 282, 2-10 (2019).

17. Li, L., Zhang, Q., Huang, D. A review of imaging techniques for plant phenotyping. Sensors (Switzerland). 14, 20078-20111 (2014).

18. Gosa, S.C., Lupo, Y., Moshelion, M. Quantitative and comparative analysis of whole-plant performance for functional physiological traits phenotyping: New tools to support pre-breeding and plant stress physiology studies. Plant Science. 282, 49-59 (2019).

19. Araus, J.L., Cairns, J.E. Field high-throughput phenotyping: the new crop breeding frontier. Trends in Plant Science. 19, 52-61 (2014).

20. Ito, V.C., Lacerda, L.G. Black rice (Oryza sativa L.): A review of its historical aspects, chemical composition, nutritional and functional properties, and applications and processing technologies. Food Chemistry. 301, 125304 (2019).

21. Anjum, S.A. et al. Morphological, physiological and biochemical responses of plants to drought stress. African Journal of Agricultural Research. (2011).

22. Halperin, O., Gebremedhin, A., Wallach, R., Moshelion, M. High-throughput physiological phenotyping and screening system for the characterization of plantenvironment interactions. The Plant Journal. 89, 839850 (2017).

23. Yaaran, A., Negin, B., Moshelion, M. Role of guard-cell $A B A$ in determining steady-state stomatal aperture and prompt vapor-pressure-deficit response. Plant Science. 281, 31-40 (2019). 
24. Dalal, A., Attia, Z., Moshelion, M. To produce or to survive: how plastic is your crop stress physiology? Frontiers in Plant Science. 8, 2067 (2017).

25. Araus, J.L., Kefauver, S.C., Zaman-Allah, M., Olsen, M.S., Cairns, J.E. Translating High-Throughput Phenotyping into Genetic Gain. Trends in Plant Science. 23, 451-466 (2018).

26. Ghanem, M.E., Marrou, H., Sinclair, T.R. Physiological phenotyping of plants for crop improvement. Trends in Plant Science. 20, 139-144 (2015).

27. Sinclair, T.R. et al. Pot binding as a variable confounding plant phenotype: theoretical derivation and experimental observations. Planta. 245, 729-735 (2017). 